\title{
Cost of best-practice primary care management of chronic disease in a remote Aboriginal community
}

\begin{abstract}
Andrew P
Gador-Whyte

BMedSc

Research Student ${ }^{1}$

John Wakerman

MBBS, MTH, HonDSc

Director, and Chief

Investigator ${ }^{2}$

David Campbell

PostgradCertManagement,

MSc, BSC

PhD Candidate

Sue Lenthall

RN, BT, MPH

Senior Lecturer

Janet Struber

BPthy, GradCertHSM, MHS

Senior Research Fellow

Alex Hope

BA, MBChB

Public Health Medical Officer

Colin Watson

RN, MTH, PhD

Lecturer in Nursing ${ }^{4}$

1 Centre for Remote Health, Alice Springs, NT.

2 Centre of Research

Excellence in Rural and

Remote Primary

Health Care,

Bendigo, VIC.

3 Aboriginal Medical Services Alliance of the Northern Territory,

Alice Springs, NT.

4 Charles Darwin University,

Alice Springs, NT.

john.wakerman@

flinders.edu.au

MJA 2014 200: 663-666 doi: 10.5694/mjal3.11183

he health of Australian Aboriginal and Torres Strait Islander people is very poor, and their access to primary care is inadequate, especially in remote areas. ${ }^{1-3}$ Prevalence of and mortality from type 2 diabetes (hereafter diabetes) and chronic kidney disease (CKD) are extremely high in remote Aboriginal communities in the Northern Territory. ${ }^{4}$ Chronic disease is responsible for $80 \%$ of the mortality gap between Indigenous and nonIndigenous Australians, with diabetes accounting for $12 \% .{ }^{5}$ For all Indigenous Australians, the self-reported prevalence of diabetes is more than three times, and the incidence of end-stage CKD six times, the non-Indigenous rates. ${ }^{3}$ In remote Aboriginal communities in the NT, CKD prevalence is up to $25 \%$ of the population, 4 or $46 \%$ of adults. ${ }^{6}$ Diabetes prevalence is in the order of $13 \% 7$ to $16 \%^{4}$ of the population, or up to $29 \%$ of adults. ${ }^{6}$ Both conditions are major contributors to cardiovascular disease, the greatest single contributor to the mortality gap. 5

The origins of the chronic disease epidemic in Indigenous populations and, more broadly, the health gap are complex, 3,6 and actions addressing both the social determinants of health ${ }^{3,4,7,8}$ and systematic primary clinical care 6,9 are needed. In this study, we focused on the need for systematic clinical care, which is essential to reduce complications and delay the progression of diabetes and CKD. 6,9

The rate of avoidable hospitalisations, a key marker of access to and effectiveness of primary care, is highest in remote communities. ${ }^{10}$ Australian and international evidence shows strong primary care systems result in better health outcomes, lower rates of avoidable hospitalisations, avoidance of dialysis, and significant cost savings. ${ }^{9-13}$ Many hospitalisations for complications of diabetes and CKD can be avoided through systematic primary care treatment, with significant medical cost savings. ${ }^{10,13-15}$ However,
\end{abstract}

\section{Abstrac}

Objective: To estimate the cost of completing all chronic care tasks recommended by the Central Australian Rural Practitioners Association Standard Treatment Manual (CARPA STM) for patients with type 2 diabetes and chronic kidney disease (CKD).

Design and setting: The study was conducted at a health service in a remote Central Australian Aboriginal community between July 2010 and May 2011. The chronic care tasks required were ascertained from the CARPA STM. The clinic database was reviewed for data on disease prevalence and adherence to CARPA STM guidelines. Recommended tasks were observed in a time-and-motion study of clinicians' work. Clinicians were interviewed about systematic management and its barriers. Expenditure records were analysed for salary and administrative costs.

Main outcome measures: Diabetes and CKD prevalence; time spent on chronic disease care tasks; completion of tasks recommended by the CARPA STM; barriers to systematic care identified by clinicians; and estimated costs of optimal primary care management of all residents with diabetes or CKD.

Results: Projected annual costs of best-practice care for diabetes and CKD for this community of 542 people were $\$ 900792$, of which $\$ 645313$ would be met directly by the local primary care service. Estimated actual expenditure for these conditions in 2009-10 was $\$ 446585$, giving a projected funding gap of $\$ 198728$ per annum, or $\$ 1733$ per patient. High staff turnover, acute care workload and low health literacy also hindered optimal chronic disease care.

Conclusion: Barriers to optimal care included inadequate funding and workforce issues. Reduction of avoidable hospital admissions and overall costs necessitates adequate funding of primary care of chronic disease in remote communities.

there are few estimates of the resources required to sustain primary care management of these conditions.

Particular difficulties for remote primary care include isolation, long distances to services, understaffing and a high turnover of clinical staff. ${ }^{16}$ Remote health care also requires special skills in chronic disease management, acute care, public health and intercultural communication. ${ }^{13,14,16,17}$

Our study was initiated by an Aboriginal community controlled remote health service because, despite its own perceptions of competent staff and clinical systems, staff still struggled to satisfy existing clinical protocols within given resources. The service received funding primarily from the then federal Office for Aboriginal and Torres Strait Islander Health and the then NT Department of Health and Families. Other sources included Medicare and a small university research grant. The aim of our study was to estimate the resources required by a remote health service to optimally manage diabetes and
CKD - with adherence to the local Central Australian Rural Practitioners Association Standard Treatment Manual (CARPA STM) guidelines ${ }^{15}$ and with full population coverage.

\section{Methods}

The study took place between July 2010 and May 2011 in a remote Central Australian community. The methods were adapted from the models of care health service planning approach ${ }^{18}$ and an earlier NT cost study. ${ }^{10}$ Using the models of care approach, Segal and colleagues ${ }^{18}$ began with local bestpractice protocols and estimated the staff time required to complete each of the recommended tasks for a given population. The population health status was assessed and the best-practice health care tasks required for that population were defined for each health profession involved. The time required per patient per year for each task was then determined, and the health care staffing resources then calculated for the population. Zhao and colleagues ${ }^{10}$ 
used an analogous approach to estimate the total operating costs of a "reasonably efficient" remote Aboriginal health service. Population needs were defined in terms of the CARPA STM protocols. ${ }^{15}$ The time estimate given in the definition of each linked Medicare Benefits Schedule item was used in quantifying the population's staffing needs. ${ }^{10}$

The target population was resident adults ( $>18$ years of age) with diabetes or CKD. Best practice was defined in terms of the CARPA STM protocols. ${ }^{15}$ These protocols are used by all NT primary care providers: government clinics and Aboriginal community controlled health services. ${ }^{19}$ The tasks recommended by the CARPA STM include history taking, health promotion, education, physical examination, biochemical investigations and prescription of medication. ${ }^{15}$

First, prevalence of diabetes and CKD was estimated using the health service electronic health record, which represents not only patients presenting for diabetes and CKD management but also those identified in community screening. Prevalence at multiple levels of severity was estimated for both conditions. For CKD, levels of severity were stages 1-5 (estimated glomerular filtration rate range, $>90$ [stage 1] to $<15 \mathrm{~mL} / \mathrm{min} / 1.73 \mathrm{~m}^{2}$ [stage 5]), with early CKD defined as stages 1-3. For diabetes, early was defined as non-insulin-treated and advanced was defined as insulin-treated. This enabled us to calculate, at the community level, the total annual number of diabetes and CKD care tasks recommended by the clinical protocols.

Second, semistructured interviews informed two aspects of the study. Clinicians were asked to estimate the time required for each protocol task, as well as any unremunerated out-of-hours time essential for the chronic disease management program. Clinicians' descriptions of their role in chronic disease care helped to structure the final staff-funding model and also to identify other barriers to bestpractice care.

Third, the daily work and chronic disease consultations undertaken by a cross-section of clinicians were directly observed in a detailed timeand-motion study. We measured the time taken by each clinician for each task defined by the relevant CARPA
1 Estimated 2009-10 and projected annual costs* for the primary care management of type 2 diabetes and chronic kidney disease in a remote Aboriginal community

Annual costs (\$)

\begin{tabular}{|c|c|c|}
\hline \multirow[b]{2}{*}{ Category } & \\
\hline & 2009-10 & Projected \\
\hline \multicolumn{3}{|l|}{ Remote health centre costs } \\
\hline Clinical staff & 228906 & 340392 \\
\hline Remote area nurses and Aboriginal health workers & 62011 & 68592 \\
\hline General practitioners & 38799 & 74439 \\
\hline Chronic disease educators and visiting dietitians & 128096 & 197362 \\
\hline Administrative staff & 76383 & 108507 \\
\hline Medical supplies & 4079 & 5795 \\
\hline Other operating costs & 137217 & 190618 \\
\hline Maintenance & 1240 & 1762 \\
\hline Vehicles & 27758 & 35123 \\
\hline Other supplies & 1635 & 2322 \\
\hline Other administrative (including computers, power) & 106584 & 151411 \\
\hline Total & 446585 & 645313 \\
\hline Direct costs to federal and NT governments ${ }^{\dagger}$ & 234424 & 255479 \\
\hline Grand total & 681008 & 900792 \\
\hline $\begin{array}{l}\text { NT = Northern Territory. *Full coverage and full adherence to } \\
\text { optometry, non-vehicle and vehicle capital costs, and medica } \\
\text { under s } 100 \text { of the National Health Act } 1953 \text { (Cwlth). }\end{array}$ & $\begin{array}{l}\text { D. † Includes } \\
\text { rovided to Ir }\end{array}$ & $\begin{array}{l}\text { al (NT), and } \\
\text { ous patients }\end{array}$ \\
\hline
\end{tabular}

STM protocol. We then used a combination of these observations and interview data to estimate an average time for each item (such as measuring blood pressure or discussing diet). Supplies were itemised for each protocol and costed using the clinic's supply orders or representative prices.

Fourth, financial records provided clinicians' wage rates and administrative fixed costs. The wage rates, together with the estimates of timeper-task and annual community care requirements, were used to calculate the total cost of clinical staffing for primary care of diabetes and CKD.

We considered costs that, while nonclinical, are part of the overall cost footprint of diabetes and CKD care. Using data from direct observation of clinicians' workdays, we estimated the proportion of total clinical time spent on diabetes and CKD. This proportion was then applied in a top-down fashion to the health service's non-clinical costs, so that part of the cost of clerical staff and clinic vehicles was attributed to diabetes and CKD. These data collection and cost estimation methods are summarised in the Appendix (online at mja.com.au).

Finally, we compared the estimated 2009-10 expenditure on diabetes and CKD with the projected annual expenditure using our best-practice model, reflecting full protocol compliance. For this, we assessed the level of clinicians' adherence to the guidelines using the One21seventy health record audit tool, which is widely accepted in Indigenous health services in Australia and is based on the CARPA STM protocols. ${ }^{20}$ Per capita figures were calculated as total staff hours required for tasks specific to each group (eg, staff hours per patient per week to manage insulin treatment) divided by the number of patients with CKD or diabetes.

We distinguished between costs met out of the health service budget and expenses met directly by the federal and NT governments and other agencies. Examples of the latter are medications provided free to Indigenous patients under s 100 of the National Health Act 1953 (Cwlth), and visiting allied health services. The increased cost of such items in our model of care was separated from any additional funding needed by the health service to provide that model.

For comparison with the study by Zhao et al we adjusted the 2003-04 NT-wide figures for inflation occurring between financial years 2003-04 and 2009-10 (using the Consumer Price Index for health care in Darwin), and only included comparable aspects of 
2 Total projected annual costs per patient* for the primary care management of type 2 diabetes and chronic kidney disease (CKD) in a remote Aboriginal community, by stage of disease and comorbidity

Cost, by severity of CKD (\$)

\begin{tabular}{|c|c|c|c|}
\hline \multirow[b]{2}{*}{ Condition and case type } & \\
\hline & No CKD or stage 1-3 & Stage 4 & Stage $5^{\dagger}$ \\
\hline \multicolumn{4}{|l|}{ CKD without diabetes } \\
\hline New cases & 4345 & 4547 & 5200 \\
\hline \multicolumn{4}{|l|}{ Existing cases } \\
\hline Without high cardiovascular risk & 4062 & 4175 & 5042 \\
\hline With high cardiovascular risk & 4125 & - & - \\
\hline \multicolumn{4}{|l|}{ Non-insulin-treated diabetes } \\
\hline New cases & 4428 & 4601 & 5252 \\
\hline \multicolumn{4}{|l|}{ Existing cases } \\
\hline Without high cardiovascular risk & 4133 & 4228 & 5093 \\
\hline With high cardiovascular risk & 4196 & - & - \\
\hline \multicolumn{4}{|l|}{ Insulin-treated diabetes } \\
\hline New cases & 14417 & 14590 & 15241 \\
\hline \multicolumn{4}{|l|}{ Existing cases } \\
\hline Without high cardiovascular risk & 14122 & 14218 & 15083 \\
\hline With high cardiovascular risk & 14185 & - & - \\
\hline
\end{tabular}

our study (eg, insulin treatment was excluded). ${ }^{10,21}$

Approval was received from the remote community's health board and the Central Australian Human Research Ethics Committee (ref: 2010.08.07). Written consent was obtained from all individual participants. Verbal and written feedback was provided to the health board at the conclusion of the study.

\section{Results}

In this remote community with a population of 542, 519 (96\%) were Aboriginal. ${ }^{22}$ Of the 297 adults, 74 (25\%) had diabetes and 86 (29\%) had CKD; 45 (15\%) had both conditions. Thirty-one adults (10\%) had CKD stages $3-5$. Of the 74 adults with diabetes, $13(18 \%)$ were prescribed insulin.

The clinic was staffed by four Aboriginal health workers (AHWs), three remote area nurses (RANs), a general practitioner, a chronic disease educator (CDE) and an exercise physiologist. Twelve staff interviews were completed, and 14 workdays and seven clinical consultations were directly observed.

Quarterly chronic disease checks with an AHW or RAN were recommended for most patients with diabetes or CKD. A GP reviewed clinical findings and investigations, prescribed medication where necessary, and oversaw treatment planning. A full-time CDE educated individual patients and groups and managed insulin treatment. Interviewed staff identified education as crucial for patients' control of their chronic disease. Locating patients in the community and delivering and explaining their medications were also a significant part of clinicians' work. According to the medical record audit, $75 \%$ of all recommended protocol tasks for diabetes management and 79\% for CKD were completed in 2009-10. Based on 2009-10 staffing, our model results in $29 \%$ of total clinical hours being used for diabetes and CKD care.

The total projected annual primary care cost of clinical management of diabetes and CKD management with full adherence to the protocols for all patients would be $\$ 900792$ (Box 1). On average, the annual cost per patient would be $\$ 7856$, ranging from $\$ 4062$ (for people with previously diagnosed early CKD without diabetes and without high cardiovascular risk) to $\$ 15241$ (for people with stage 5 CKD and advanced diabetes, with a recent diagnosis of either condition) (Box 2). Including only the costs met through the health service-allocated budget, the projected cost to the centre would be $\$ 645313$, or $\$ 5628$ per patient on average, based on estimated total annual direct clinical staff hours specifically for diabetes and CKD of 4226 hours, or 2.37 full-time equivalents. (The difference between the cost to the centre and the total projected annual cost consists of costs met directly by other sources, including the federal and NT governments, rather than through the health service budget.) Expenditure in 2009-10 by the remote health service for diabetes and CKD care was estimated at $\$ 446585$, or $\$ 3895$ per patient (Box 1). The health service would thus require an additional \$198728 (ie, an additional \$1733 per patient) for best-practice management of diabetes and CKD. Overall funding received by the health centre in 2009-10 was \$3413 per patient.

Interviews with staff identified a number of other barriers to optimal care. The high turnover of RANs necessitated frequent intensive orientation and teaching for new staff. The clinic was also rarely fully staffed. On-call night work, cross-cultural communication and cultural differences can present difficulties for RANs. AHWs may face a conflict of duties and values between the community and the health centre and a heavy responsibility in the role. A high acute caseload and the reticence of many patients to attend checks, for reasons including low health literacy and long waiting times, also hinder adequate management of chronic disease. There is a perceived need to allow more time for clinicians and patients to build relationships and for community-building work.

\section{Discussion}

Our study identified a high prevalence of diabetes and CKD in a remote community. As this is the community's only clinic and the community health screening coverage is high, the clinicbased prevalence approximates population prevalence and is consistent with other remote community studies. 4,13

This service is relatively well funded compared with other NT primary care services. Expenditure in 2009-10 was \$3413 per capita per annum. The mean per capita figure for NT Government remote clinics for 2011-12 was \$2329. This service has a higher than average compliance with the protocols for CKD and diabetes $(77 \%$, compared with the 
territory-wide figure of $73 \%$ for clinics participating in the One21Seventy audit). In response to low health literacy and patient mobility, the clinicians engage in very active outreach, health education and opportunistic screening. Most remote communities do not employ a CDE or exercise physiologist.

Despite these positive factors, 2009-10 expenditure was insufficient to provide optimal management by a margin of $44 \%$, or an average of $\$ 1733$ per patient with diabetes or CKD. Quantitative data were consistent with clinicians' perceptions at interview that additional staff members were required to work with the CDE and AHWs to adequately meet community needs. Additional staff could increase the crucial quarantining of AHWs' and RANs' chronic disease care time from acute care time, possibly contributing to enhanced retention of staff. It would also allow greater allied health coverage, increasing exposure of patients to consistent health messages, and additional informal staff education.

The costs calculated in our study were higher than the inflation-adjusted (2003-04 to 2009-10) NT remote community estimates produced by Zhao et al. ${ }^{10,21}$ Possible reasons include the conservative estimates by Zhao et al of clinical time needed in remote health services for chronic disease care and staff training, and the higher administrative and salary costs at the studied remote clinic compared with those used in the earlier study. ${ }^{10}$

Strong primary care systems result in better health outcomes and significant cost savings. $9,11-13$ There is a strong argument for adequate funding of primary care services like the subject of our study. Our findings also highlight the lack of benchmarks that might indicate appropriate levels of funding to meet community needs and provide cost-effective care.

The strength of this study is its rigour and comprehensiveness, using multiple data sources (qualitative and quantitative). However, the methods are resource intensive and replication at other locations or with other disease groups would be expensive. Other limitations include the fact that costs were kept constant in our modelling. It is likely that unit and, possibly, fixed costs will increase as 100\% community coverage and adherence to protocols is approached. It may be that $100 \%$ coverage and compliance is uneconomical.

Limitations to generalising these findings to other remote Indigenous health centres are the wide variation in transport costs, the availability of allied health care, and the feasibility of indicated insulin treatment given limited staffing resources. The small sample size also means that caution must be taken in generalising the results.

Nevertheless, this community is not unrepresentative of many remote Indigenous communities, and our study provides the best available evidence about the funding gaps for optimal management of two prevalent chronic diseases in such communities. It documents a significant shortfall even in a relatively well funded and effective primary care service, close to the regional centre. We would expect that the documented shortfall is a conservative estimate. These findings should inform funding bodies in their allocation of health resources for remote Indigenous communities. It may also be of use to this and other remote communities in advocating for their health care needs.

Competing interests: No relevant disclosures.

Received 13 Sep 2013, accepted 08 Jan 2014.

1 National Rural Health Alliance. Australia's health system needs re-balancing: a report on the shortage of primary care services in rural and remote areas. Canberra: NRHA, 2011. http:// www.ruralhealth.org.au/sites/default/files/ documents/nrha-policy-document/position/ pos-full-complementary-report-27-feb-11.pdf (accessed Jan 2014)

2 Vos T, Barker B, Begg S, et al. Burden of disease and injury in Aboriginal and Torres Strait Islander Peoples: the Indigenous health gap. Int J Epidemiol 2009; 38: 470-477.

3 Australian Institute of Health and Welfare. Australia's health 2012. Canberra: AlHW, 2012. (AlHW Cat. No. AUS 156.)

4 Zhao Y, Connors C, Wright J, et al. Estimating chronic disease prevalence among the remote Aboriginal population of the Northern Territory using multiple data sources. Aust N Z J Public Health 2008; 32: 307-313.

5 Australian Institute of Health and Welfare. Contribution of chronic disease to the gap in mortality between Aboriginal and Torres Strait Islander people and other Australians. Canberra: AlHW, 2011. (AlHW Cat. No. IHW
48.) http://www.aihw.gov.au/publicationdetail/?id=10737418878 (accessed Oct 2012).

6 Hoy WE, Davey RL, Sharma S, et al. Chronic disease profiles in remote Aboriginal settings and implications for health services planning. Aust N Z J Public Health 2010; 34: 11-18.

7 McDermott R, O'Dea K, Rowley K, et al. Beneficial impact of the homelands movement on health outcomes in central Australian Aborigines. Aust N Z J Public Health 1998; 22: 653-658.

8 Carson B, Dunbar T, Chenhall RD, Bailie R, editors. Social determinants of Indigenous health. Sydney: Allen \& Unwin, 2007: xix-xxii.

9 You J, Zhao Y, Beaver, C, et al. End-stage renal disease in the Northern Territory: current and future treatment costs. Med J Aust 2002; 176: 461-465.

10 Zhao Y, Hanssens P, Byron P, Guthrdige S. Cost estimates of primary health care activities for remote Aboriginal communities in the Northern Territory. Darwin: NT Department of Health and Community Services, 2006. http:// digitallibrary.health.nt.gov.au/prodjspui/ bitstream/10137/59/3/Primary_health_care_ costs.pdf (accessed Jan 2014).

11 McDermott R, Segal L. Cost impact of improved primary level diabetes care in remote Australian Indigenous communities. Aust J Prim Health 2006; 13: 124-130.

12 Starfield B, Shi L, Macinko J. Contribution of primary care to health systems and health. Milbank Q 2005; 83: 457-502.

13 Hoy WE, Wang Z, Baker PR, et al. Reduction in natural death and renal failure from a systematic screening and treatment program in an Australian Aboriginal community. Kidney Int Suppl 2003; (83): S66-S73.

14 Si D, Bailie R, Weeramanthri T. Effectiveness of chronic care model-oriented interventions to improve quality of diabetes care: a systematic review. Prim Health Care Res Dev 2008; 9: 25-40.

15 Central Australian Rural Practitioners Association. CARPA standard treatment manual. 5th ed. Alice Springs: CARPA, 2010.

16 Wakerman J. Defining remote health. Aust $J$ Rural Health 2004; 12: 210-214.

17 Thompson N. The health of Indigenous Australians. Melbourne: Oxford University Press, 2004

18 Segal L, Dalziel K, Bolton T. A work force model to support the adoption of best practice care in chronic diseases - a missing piece in clinical guideline implementation. Implement Sci 2008; 3: 35.

19 Chaseling M, Mentha R, Davey C. CARPA Standard treatment manual 4th edition evaluation report. Alice Springs: Centre for Remote Health, 2008.

20 One21seventy. Clinical audit tools. Vascular and metabolic syndrome management for type 2 diabetes, coronary heart disease, chronic heart failure, chronic kidney disease and hypertension, version 3.0. Brisbane: One21seventy, 2009.

21 Australian Bureau of Statistics. Consumer Price Index, Australia, Mar 2011. Canberra: ABS, 2011. (ABS Cat. No. 6401.0.)

22 Australian Bureau of Statistics. National Aboriginal and Torres Strait Islander Health Survey, 2004-05. Canberra: ABS, 2006. (ABS Cat. No. 4715.0.) http://www.abs.gov.au/ ausstats/abs@.nsf/mf/4715.0 (accessed Jan 2014). 